\title{
Extractivismo, género y energía eólica en el municipio de Zacatecas. ¿Desarrollo para quién?
}

\author{
Verónica Vázquez García* \\ Dulce María Sosa Capistrán**
}

Resumen. Este artículo examina los impactos y beneficios de la transición energética desde la perspectiva de género. Tomando el caso del Parque Eólico La Bufa ubicado en el municipio de Zacatecas, se propone responder a la siguiente pregunta: ¿qué beneficios reciben las mujeres de la llegada de la industria eólica a su territorio? Para recabar la información se utilizó una metodología mixta que involucró la realización de cuestionarios, entrevistas a profundidad, grupos focales y ejercicios de devolución de resultados ante tomadores de decisiones. A partir de los datos se demuestra que el modelo extractivista característico del neoliberalismo verde reproduce las jerarquías de género en el sentido de que las mujeres, principales transformadoras de energía desde sus hogares, están excluidas de procesos de toma de decisiones, uso del territorio, reparto de beneficios y aprovechamiento de energía eólica.

Palabras clave: Parque Eólico La Bufa, perspectiva de género, toma de decisiones, neoliberalismo verde, extractivismo.

* Profesora investigadora, Colegio de Postgraduados, Campus Montecillo, México.

** Estudiante del Doctorado en Estudios Latinoamericanos en Territorio, Sociedad y Cultura, Universidad Autónoma de San Luis Potosí, México. 


\section{Extractivism, gender and wind energy in the municipality of Zacatecas. Development for who?}

Abstract. This article examines the impacts and benefits of energy transition from a gender perspective. It takes up the case of «La Bufa» Wind Park located in the municipality of Zacatecas, in order to answer the following question: What benefits are obtained by women with the arrival of the wind industry in the region? Information was gathered using a mixed methodology which involved the carrying out of questionnaires, in-depth interviews, focus groups, and seeking feedback from decision-makers. The data showed that the extractivist model characteristic of «green neoliberalism» reproduces gender hierarchies, in the sense that women, the principal users of energy in their homes, are excluded from the decision-making processes, use of land, sharing of benefits and utilization of wind energy.

Keywords: «La Bufa» Wind Park, gender perspective, decision-making, green neoliberalism, extractivism. 


\section{Introducción}

La industria eólica comenzó a adquirir relevancia en México a partir de los 1990. El sitio de mayor recepción de esta fuente renovable de energía (FRE) ha sido el Istmo de Tehuantepec, Oaxaca, región que actualmente tiene capacidad instalada para generar 2 mil $695 \mathrm{MW}$ que representan 82\% del total de energía eólica a escala nacional, misma que se produce en 24 parques con alrededor de mil 400 aerogeneradores (García-Torres, 2018; Avilés, 2018). Otros estados donde la energía eólica ha empezado a llegar en proporciones menores son Baja California, Tamaulipas, Zacatecas, Sinaloa, Hidalgo, Veracruz, Puebla y Yucatán. La Asociación Mexicana de Energía Eólica (AMDEE) proyecta que dichos estados podrían aportar hasta 10 mil MW de capacidad al parque eléctrico nacional (AMDEE, 2018).

La mayor parte de los estudios sobre la llegada de esta FRE a México han sido realizados en el Istmo de Tehuantepec. Se han identificado las siguientes afectaciones ambientales producto de la adopción de energía eólica: cambios en el paisaje y fragmentación del hábitat; deforestación; muerte de aves y murciélagos por colisión; contaminación de suelo y agua por derrame de aceite y vertimiento de concreto; disminución de poblaciones pesqueras por ruido, residuos o vibraciones. Por su parte, los impactos sociales son los siguientes: falta de información sobre los proyectos eólicos; desarticulación de la vida social al interior de familias y comunidades; pérdida de acceso y abandono de espacios productivos; incremento en la inseguridad alimentaria. Entre los beneficios está el cobro monetario por concepto de regalías o renta de terrenos (Sánchez, 2008; Castillo, 2011; Juárez-Hernández y León, 2014; Nahmad et al., 2014; López, 2015; Levy et 
al., 2015; Diego, 2014, 2015, 2016; Dunlap, 2018). Gay-Antaki (2016) y García Torres (2018) demuestran que cada una de estas afectaciones tienen impactos diferenciados en mujeres y hombres dado que su acceso, uso y control de recursos naturales está marcado por construcciones sociales de género.

Como puede verse, la transición energética, tan necesaria para combatir el cambio climático, representa un importante reto para el desarrollo. Este artículo se propone responder a la siguiente pregunta, tomando el caso específico del Parque Eólico La Bufa ubicado en el municipio de Zacatecas: ¿qué beneficios reciben las mujeres de la llegada de la industria eólica a su territorio? Se argumenta que el modelo extractivista reproduce las jerarquías de género en el sentido de que las mujeres, principales transformadoras de energía desde sus hogares, están excluidas del proceso de toma de decisiones, del uso de los espacios en disputa y de la innovación tecnológica inherente a la industria eólica.

\section{Propuesta conceptual: energía eólica, neoliberalismo verde y jerarquías de género}

El extractivismo se refiere a la apropiación de un recurso natural a gran volumen o alta intensidad para obtener productos orientados a la exportación como materias primas sin procesar, o con un procesamiento mínimo. El extractivismo tiene dos características principales: la sobreexplotación de los recursos naturales y su orientación exógena o exportadora. La primera abarca una tasa y ritmo de extracción de recursos superior al de los tiempos biológicos, químicos y geológicos de reposición natural, mientras que la segunda se refiere a la dependencia macroeconómica estructural, 
pues el extractivismo se concibe como un medio para proveer materias primas a otras economías (Machado, 2015). Los precios de los productos extraídos rara vez toman en cuenta los daños socioambientales ocasionados por la sobreexplotación, por lo que la actividad comercial constituye en sí misma un intercambio ecológico desigual. Los países en vías de desarrollo se especializan en la exportación de materias primas, mientras que las economías desarrolladas les agregan valor, convirtiendo a los primeros en economías de enclave, dependientes de insumos y tecnologías del exterior (Gudynas, 2013).

Lo interesante de la energía eólica es que se enmarca dentro de la propuesta de desarrollo sustentable porque el viento es recurso renovable que no puede ser agotado; su aprovechamiento no implica sobreexplotación. De hecho, las FRE (sol, viento, olas y mareas, geotermia, agua y biocombustibles) se han convertido cada vez más en la pieza clave de la transición energética gracias a su potencial para reducir la dependencia de combustibles fósiles (no renovables) y crear empleos «verdes» que ayuden a restaurar y preservar el medio ambiente. También se ha argumentado que la energía eólica tiene el potencial de suministrar energía eléctrica a comunidades rurales y marginadas tanto para México (CNDPI, 2013) como para otras partes del mundo (Corscadden et al., 2012; Simcock, 2014; Okkonen y Lehtonen, 2016). Dicho potencial depende del modelo de gobernanza ambiental adoptado. En México predomina el neoliberalismo verde, entendido como un modelo económico que privilegia al mercado como eje central de la economía. En su relación con la naturaleza, el neoliberalismo verde involucra la privatización y comercialización de los recursos naturales para beneficio de empresas multinacionales (Durand et al., 2019). Honty y Gudynas definen al neoliberalismo verde como 
un grupo de alternativas que se enfocan en reformar las estrategias de desarrollo, sea por medio de ajustes o cambios dentro del capitalismo, manteniendo algunos de sus elementos centrales, tales como la necesidad de crecer económicamente o la mercantilización de la naturaleza. Postula soluciones tales como reconocer elementos y procesos de la naturaleza como bienes y servicios que se pueden comprar en el mercado (2014:27).

Desde esta perspectiva, los países en vías de desarrollo que producen energía eólica continúan en una situación de dependencia de insumos y tecnología, con la diferencia de que la materia prima extraída es el viento.

Otras iniciativas de combate al cambio climático, como Reducción de Emisiones derivadas de la Deforestación y la Degradación de los Bosques (REDD+) y los biocombustibles, también han sido catalogados como instrumentos del neoliberalismo, no sólo porque el marco utilizado para diseñarlas continúa en la dinámica de la mercantilización, sino también porque se transfiere a espacios del Sur Global la responsabilidad de reducir la emisión de gases de efecto invernadero, impidiendo que las comunidades decidan sobre la orientación de su propio desarrollo (Trench y Libert, 2019; Tejada y Rist, 2019). El neoliberalismo verde se propone como la única fórmula posible para afrontar la necesaria transición energética; sus contenidos generalmente involucran cambios tecnológicos (por ejemplo, la industria eólica) que no logran trastocar los principios de acumulación del capital y de desigualdad social que son inherentes al capitalismo (Martínez y Gómez, 2017).

Los procesos de neoliberalización no son neutrales al género. En contextos extractivistas es indispensable analizar tres condiciones que determinan la relación de las mujeres con el territorio: «a) Los Estados no 
reconocen el trabajo de las mujeres rurales; b) las mujeres rurales no tienen acceso a la tierra y no son dueñas de la misma; c) las mujeres rurales no son consultadas y, en su mayoría, no toman decisiones referentes a los usos de sus territorios» (Olivera, 2019:253). En estas condiciones, cuando el capital penetra determinado territorio, se incrementa la feminización de los cuidados, la masculinización de la toma de decisiones y la pérdida de acceso a espacios de uso tradicional por parte de las mujeres. Esto a su vez tiene impactos negativos en su salud, uso de recursos y calidad de vida. La perspectiva de género analiza los impactos diferenciados del extractivismo, así como el sistema sobre el cual se asientan dichas jerarquías (Composto y Navarro, 2014), porque lo que está de por medio es «la imposición de un determinado modelo de desarrollo colonial y (...) patriarcal dirigido a satisfacer los intereses de la clase capitalista global» (García-Torres, 2018:7).

Partiendo de esta base conceptual, el presente artículo estudia los impactos y beneficios obtenidos de la adopción de FRE en el municipio de Zacatecas en relación con las jerarquías de género y el uso doméstico de diferentes fuentes de energía. Específicamente se revisará el contexto del Parque Eólico La Bufa (PEB) y las implicaciones que éste ha tenido para las mujeres y hombres de comunidades rurales y marginadas cercanas al parque. El argumento es doble. En primera instancia se identifican y analizan los contenidos de justicia social de la transición energética del municipio. Segundo, se estudian las dimensiones de género de esta forma de capitalismo verde en torno a los siguientes temas: acceso de las mujeres a información sobre el parque; cambios en su uso del territorio; participación en la renta de la tierra; acceso a fuentes de energía doméstica que beneficien su salud, aligeren su carga de trabajo y contribuyan a su calidad de vida. 


\section{Zona de trabajo y metodología}

La adopción de energías renovables en México ha venido acompañada de reformas legislativas tales como la Ley del Servicio Público de la Energía (1994), que creó las modalidades de autoabastecimiento, producción independiente y cogeneración de energía con la finalidad de permitir la inversión privada en el sector; hasta el momento han predominado las dos primeras (Martínez et al., 2018). En 2008 se promulgó la Ley para el Aprovechamiento de Energías Renovables, que abrió el mercado a la inversión privada al declarar a la generación de electricidad como una actividad de utilidad pública que privilegia a los parques eólicos sobre otros usos del suelo (Diego, 2015). El neoliberalismo verde implica la readecuación de los marcos legales a las necesidades del capital, y esto es precisamente lo que ha sucedido en México con la energía eólica.

El estado de Zacatecas apenas cuenta con dos parques eólicos: Vientos del Altiplano y el PEB. El primero fue instalado en los municipios de Mazapil y Villa de Cos, y tiene capacidad para generar 100 MW. El segundo es el de interés para la presente investigación. Su permisionario es México Power Group (MPG), compañía mexicana constituida en 1979, especializada en energía eólica y solar que actualmente está desarrollando proyectos en nueve estados, «cubriendo prácticamente todas las áreas de viento significativo identificado en el país» (MPG, 2014). El PEB abarca los municipios de Zacatecas, Guadalupe, Genaro Codina y Villanueva. La empresa inició gestiones desde 2009 y consiguió la firma de un contrato de renta de terrenos ejidales y propiedad privada por 30 años. La proveedora de tecnología es Gamesa, empresa que junto con Acciona concentran 80 por ciento del suministro de energía eólica en México (Martínez et al., 2018). 
La extensión del PEB es de 11 mil hectáreas, con la idea de ampliarse hasta 14 mil para un total de 90 aerogeneradores de dos MW cada uno. El parque genera 180 MW, más que suficiente para abastecer cerca de 200 mil viviendas que probablemente podrían satisfacer las necesidades de energía de los 146 mil 147 habitantes reportados por el Instituto Nacional de Estadística y Geografía (INEGI) en 2015 para la ciudad de Zacatecas. Sin embargo, la modalidad de autoabastecimiento establece que la energía producida por concesionarios debe ser suministrada a clientes industriales. La energía que se genera en el PEB se destina a las plantas de autos Volkswagen ubicadas en Guanajuato y Puebla.

Algo similar sucede en el Istmo de Tehuantepec, donde Cementos Mexicanos (CEMEX), Wal-Mart y Bimbo figuran entre las principales usuarias de la energía eólica. Las comunidades rurales donde ésta se produce son presentadas como beneficiarias del proyecto por los ingresos que reciben por rentar la tierra (Howe y Boyer, 2016). En ese sentido, el negocio eólico es redituable tanto para las empresas generadoras de energía como para sus clientes. Las primeras tienen incentivos fiscales, contratos seguros de suministro de energía y acceso a financiamiento de organismos internacionales. Las segundas reciben energía a precios competitivos en comparación con el sector residencial, servicios y pequeñas empresas (Martínez y Gómez, 2017).

Los datos fueron recabados a través de una estrategia metodológica mixta. Se seleccionaron cinco localidades circundantes al PEB que cumplieran con la característica de ser rurales y marginadas con el fin de evaluar los aportes de la energía eólica al desarrollo social de este tipo de comunidades (figura 1). Las localidades fueron clasificadas en urbanas (colonia Alma Obrera), periurbanas (El Orito) y rurales (Ojo de Agua de Meléndez, Picones y Benito Juárez) por dos criterios principales. El 
primero es el material de las viviendas. En el medio urbano y periurbano las construcciones son más homogéneas y predominan las casas de mampostería, mientras que en el medio rural las viviendas son más variadas; la mitad son de adobe y la otra mitad de mampostería.

\section{Figura 1}

Ubicación del Parque Eólico La Bufa y localidades de estudio

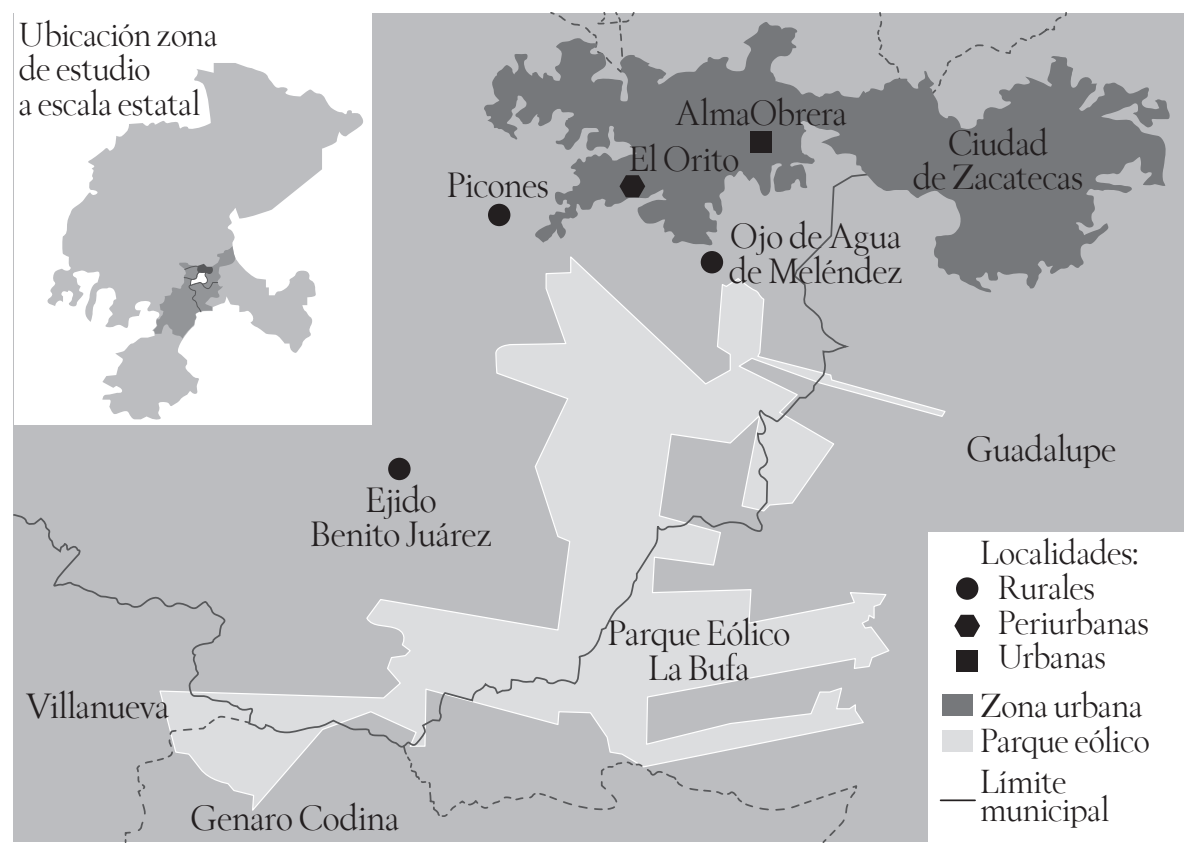

Fuente: elaborado por Rocío Martínez González con datos de INEGI (2015) y Asesoría Ambiental Integral (s/f).

El segundo criterio de clasificación fue la orientación productiva de cada localidad. En todos los hogares predomina la división sexual del trabajo que otorga a las mujeres la responsabilidad de las labores de cuidado 
y a los hombres la de proveer económicamente. Los del medio urbano son albañiles, choferes o mecánicos; algunos (sobre todo en El Orito) tienen cultivos y ganado. Las mujeres se dedican al trabajo doméstico y algunas se autoemplean como costureras, estilistas o vendiendo productos de comer y de belleza, otras son trabajadoras domésticas. En el medio rural los hombres se dedican principalmente a la producción agrícola, la ganadería (Picones y Ojo de Agua de Meléndez) y la extracción de piedra (Benito Juárez), mientras las mujeres se hacen cargo de todo el trabajo doméstico.

Se aplicaron 159 cuestionarios de manera que los tres sectores estuvieran representados: 40 en Alma Obrera; 66 en El Orito; 53 en Benito Juárez, Ojo de Agua de Meléndez y Picones. Además, se realizaron 22 entrevistas a profundidad a personas de distintas características y afiliaciones institucionales. ${ }^{1}$ También se llevaron a cabo grupos focales con mujeres de Alma Obrera, El Orito y Benito Juárez con la finalidad de profundizar en temas de interés y compartir los hallazgos de investigación. Al final del proyecto se realizaron tres ejercicios de devolución de resultados ante tomadores de decisiones en el nivel estatal y municipal con el propósito de coadyuvar al diseño de políticas energéticas con enfoque de justicia social y de género. A estas reuniones asistieron personas que participaron en el estudio quienes tuvieron oportunidad de dialogar con autoridades sobre las necesidades de energía en cada localidad.

${ }^{1}$ Algunas entrevistas fueron individuales y otras grupales. Participaron 12 mujeres y 12 hombres en total. La lista de personas incluye a personal del gobierno estatal y municipal; autoridades ejidales; operadores de partidos políticos; activistas ambientales; trabajadores de la empresa; ejidatarios de El Orito, Picones y Benito Juárez; habitantes de todas las localidades involucradas; integrantes de la Asociación Civil Inclusión Zacatecas A.C. (IZAC), la cual tiene como objetivo la inclusión social de personas en situación de vulnerabilidad mediante proyectos de empoderamiento económico a partir de tecnologías ambientalmente responsables. Para fines de confidencialidad se utilizan pseudónimos en la exposición de los resultados. 


\section{El Parque Eólico La Bufa: impactos y beneficios}

\section{Impactos diferenciados por género en el acceso y uso del territorio}

El cuestionario arrojó que una pequeña minoría de mujeres encuestadas considera haber recibido suficiente información sobre la llegada del PEB a la región (gráfica 1). Las entrevistas cualitativas dieron resultados similares. La gente tiene ideas generales o incluso erróneas del proyecto: «Son como los esos boilers solares que están metiendo (...) Ahora van a agarrar electricidad por ahí en los cerros» (Rafael Pérez, minero jubilado). El origen de la empresa lo tienen poco claro: «Creo que según esto es canadiense» (Rafael Pérez, minero jubilado). El modelo de desarrollo de la industria eólica tiene un diseño top-down ideológicamente marcado por esquemas de modernización ecológica e impulsado por instancias gubernamentales que privilegia la inversión privada y el beneficio de grandes consumidores eléctricos por encima de los intereses de la población local, la cual permanece al margen del proceso de toma de decisiones (Martínez y Gómez, 2017). Este modelo es característico del capitalismo verde que impone esquemas de reconversión productiva o conservación ambiental sin tomar en cuenta a las poblaciones que habitan el territorio (Penna-Firme, 2015).

Según José Gallardo, capacitador en energías renovables de Inclusión Zacatecas A.C., la falta de acceso a información fue intencional, porque al gobierno no le convenía que la gente supiera que la energía generada no sería aprovechada en la región:

[La energía generada] es para Volkswagen Puebla, y qué frustrante que los tengas aquí, por qué crees que el gobierno se quedó calladito, calladito, mejor 
no hablaron del parque eólico, porque toda la gente se da cuenta. Así como estamos platicando nosotros, se corre la voz de que nosotros qué beneficio tenemos (...) frustrante es la palabra. Meses pasados hicieron como publicidad de ciencia y tecnología, nada más pusieron lonas (...) y pintaron bardas publicitarias (...) tristemente el gobierno así nos tiene, con el pie en la cabeza y en el suelo, así de triste (José Gallardo, capacitador IZAC).

\section{Gráfica 1}

Porcentaje de mujeres informadas sobre la instalación del PEB

- Rural Urbana $\square$ Periurbana

100

90

80

70

60

50

40

30

20

10

0

(1)


porque «ya no van a dejar sembrar» o el espacio para hacerlo será más reducido. Una cantidad un poco mayor (23 de 159) identificaron impactos negativos en ganado o fauna silvestre que atribuyeron a la reducción de áreas de pastoreo, el alejamiento de animales o que éstos se encuentran en peligro de muerte por colisión con los aerogeneradores.

Las entrevistas cualitativas permitieron profundizar en tales temas. Algunos ejidatarios expresaron preocupación porque en esos terrenos «traemos animales, tenemos tierras de agricultura». Representantes de la empresa dijeron que no había problema porque las turbinas «iban a estar muy altas, y en realidad sí están muy altas» (Mauricio López, agricultor). Durante la construcción del parque fue necesario tomar medidas para proteger ganado y cultivos: «El proyecto puso sus guardaganados para que no se atraviesen a donde están las turbinas» (Bernardo Jiménez, empleado MPG). También se instaló «un vaquero ahí que esté vigilando que no se metan animales de otros ejidos» (Fabiola Sepúlveda, Procuraduría Agraria).

Desde la época de construcción del parque hasta el día de hoy que ya está operando, sólo los «socios» (ejidatarios que rentaron tierras) pueden ingresar al PEB. Para hacerlo deben llevar «una calcamonía y algunos los traemos en las camionetas que nos movemos». Se puede ingresar con invitados, «por decir, yo llevo mi camioneta e invito a Paula o invito a otra familia, vamos a dar la vuelta, pues me dejan entrar. Pero si va ella y su esposo y no tienen identificación, no la dejan entrar» (Mauricio López, agricultor). La persona con la autorización de ingreso se hace responsable del comportamiento de sus invitados: «Haz de cuenta que si tú vas y sacas el permiso, luego no falta quien lleve un bote, una botella, y la tire ahí. Entonces, si nosotros vamos y nos encontramos un bote o algo, pues vamos a ir sobre ti, y a ti es al que vamos a sancionar» (Hernán Lavalle, agricultor). 
El PEB ya es considerado propiedad privada y se quieren evitar «actos de vandalismo (...) no puede entrar gente extraña al proyecto» (Benjamín Jiménez, empleado MPG).

Las consecuencias de la privatización del territorio para las mujeres de El Orito y Picones no fueron previstas por nadie, como sí lo fueron los impactos en el ganado de propiedad mayormente masculina. Cada año, las mujeres de ambas localidades formaban parte de la cabalgata con dirección al cerro destinada a recabar fondos para las reinas de las fiestas patronales de ambas localidades (15 de mayo en Picones y 13 de junio en El Orito). Después de negociar, el conflicto se resolvió de manera favorable: la empresa autorizó el ingreso anual de las reinas y sus bases de apoyo, por supuesto, con previa autorización y bajo la certeza de que las normas de comportamiento serán respetadas. Aparentemente, sin embargo, el trato del personal de vigilancia no es apropiado. En uno de los ejercicios de devolución de resultados realizado al final del proyecto, una participante señaló que los ciclistas han visto restringido su acceso al PEB, y que los vigilantes «nos tratan como delincuentes».

\section{La exclusión de las mujeres en el cobro de regalías}

La decisión de rentar terrenos ejidales se tomó en el seno de la asamblea ejidal y sólo las personas con derecho agrario tuvieron posibilidad de opinar y votar. Tanto en Oaxaca como en Zacatecas, esta medida condujo al fortalecimiento del poder masculino, porque únicamente las autoridades ejidales (que por lo general son hombres) tuvieron la posibilidad de negociar con los agentes externos que llegaron con la propuesta de inversión (Gay-Antaki, 2016; García-Torres, 2018). 
Los ingresos por regalías o renta de terrenos constituyen el beneficio más importante de los parques eólicos para los ejidos (Torres y Luna, 2013). En el caso del PEB, la empresa rentó tierras de varios ejidos (Machines, El Visitador, San José de Tapias y El Orito) además de algunas superficies de pequeños propietarios. Las asambleas ejidales debían aprobar el contrato ante notario «porque pues supuestamente son actos trascendentales» (Fabiola Sepúlveda, Procuraduría Agraria). Otros ejidos, por ejemplo Benito Juárez, también fueron invitados a participar, pero los ejidatarios no aceptaron por dos motivos principales: la duración del contrato (que les pareció demasiado largo) y la incertidumbre sobre la necesidad de hacer cambios en el uso del suelo (de agrícola a industrial). La empresa tuvo que modificar el polígono para «darle la vuelta» a los ejidos que no entraron al esquema de rentas (Fabiola Sepúlveda, Procuraduría Agraria).

Con los ejidos que sí aceptaron rentar se hicieron contratos prorrogables de 30 años. Los ejidos fueron identificados de acuerdo con el polígono previamente diseñado por la empresa. La Procuraduría Agraria dio asesoría para que los contratos sean «justos, se apeguen a derecho» (Fabiola Sepúlveda, Procuraduría Agraria). En realidad, plantea la misma entrevistada, los ejidatarios no tenían mucha posibilidad de negarse y si lo hacían eran considerados enemigos del desarrollo por su edad avanzada:

Ellos [los ejidatarios] quieren seguir bajo el mismo esquema, y no se puede. Estamos hablando de tres etapas de la reforma agraria. La primera fue Lázaro Cárdenas y el reparto, la segunda fue Salinas y el Programa de Certificación de Derechos Ejidales (Procede) y la tercera es esta, en donde ahora hay que buscar la asociación, la participación, la inversión y buscar que la tierra produzca y no nada más sea por actividades primarias. Y esta tercera etapa 
es la que se resisten a entender, será porque ya son personas mayores (Fabiola Sepúlveda, Procuraduría Agraria).

El contrato estableció 4\% de regalías en relación a las ventas de energía (León, 2017). El monto es adecuado en comparación con otros países donde se paga entre 1\% y 5\% de los ingresos brutos. En el Istmo de Tehuantepec los contratos se hicieron durante la década de 1990 y los primeros años del siglo XXI, y el monto establecido en aquel entonces fue bastante más bajo - entre $0.025 \%$ y $1.53 \%$ por concepto de regalías. Sólo se encontró un parque mexicano que recibe porcentajes similares a lo que se paga en el PEB. Se trata del parque manejado por EDF Energies Nouvelles construido en 2010 en La Mata y La Ventosa que paga 3.38\% a los ejidatarios de estos ejidos oaxaqueños (CNDPI, 2013). No hay que olvidar que, desde el punto de vista neoliberal, la gestión del medio ambiente persigue la eficiencia económica donde predominan criterios de maximización de utilidades. Sin duda, la industria eólica se ha hecho más eficiente con el paso del tiempo.

El ejido El Orito tiene una extensión de 2 mil 356 hectáreas que pertenecen a 93 ejidatarios; 755 hectáreas son tierras de riego y mil 439 son de uso común. El área rentada a la empresa MPG comprende cerca de 400 hectáreas de uso común que pertenecen a 60 ejidatarios, los cuales tuvieron que constituirse como socios. La sociedad incluye sólo a las personas que tienen terrenos donde está instalado el parque. El comisariado ejidal es la persona que firma a nombre de ésta. El trato de la empresa ya no es con la asamblea ejidal, sino con la sociedad que representa a «todos los titulares de una parcela que esté en la zona» (Fabiola Sepúlveda, Procuraduría Agraria). 
A diferencia de algunos ejidos del Istmo de Tehuantepec donde el dinero llega de manera individual a cada ejidatario o ejidataria, en El Orito se hace un solo pago y el dinero se reparte al interior del ejido a partir de los siguientes criterios:

A mí me tocó en una esquina de mi parcela, pero me cruzaron toda esta la mitad de tierra para entrar a esta torre y así, entonces, según eso es el 80 y el 40. Para los que no tienen ninguna afectación, pero entran como compañeros en toda el área, en todo el terreno (...) a ellos creo que [les toca] el 40 [por ciento]. Los que tenemos afectación y antena, creo que según eso el 80 por ciento de lo que nos vayan a dar ellos (Mauricio López, agricultor).

Como ya se mencionó, en México no se reconoce el derecho de mujeres rurales a la propiedad y a la participación en la toma de decisiones referentes al uso del territorio (Olivera, 2019). De los 93 ejidatarios que conforman al ejido, sólo once (poco más de 10\%) son mujeres. Asimismo, de los 60 ejidatarios que se asociaron para rentar, únicamente seis (10\%) son de sexo femenino. Los terrenos de las otras cinco no forman parte del polígono seleccionado por la empresa. Tuvimos la oportunidad de presenciar una asamblea de socios a la cual asistieron únicamente tres de las seis mujeres que la conforman. Fue imposible conversar con ellas ya que los hijos de las mujeres también asistieron a la reunión y todo el tiempo les susurraban a sus madres lo que debían de respondernos. Según la visitadora agraria, las ejidatarias de El Orito

ya son las herederas, son las sucesoras, porque el marido ya falleció. La asamblea de El Orito es susceptible a que ellas estén, pero hay otros ejidos en 
donde las mujeres no van porque los hombres no las dejan. En El Orito, aunque participen, las ejidatarias tienen la costumbre de preguntarle al hijo mayor, porque incluso para heredar ellas siempre escogen a los hijos varones (Fabiola Sepúlveda, Procuraduría Agraria).

La falta de beneficios del PEB entre las ejidatarias se extiende al resto de las mujeres. La encuesta arrojó que sólo 15.7\% de las mujeres encuestadas consideran que el PEB trajo beneficios a sus respectivas localidades. La mayoría de las que dieron una respuesta favorable son de El Orito, y el porcentaje de las que reconocieron algún beneficio en esta localidad no supera 20\%. Las que dieron una respuesta afirmativa sólo se refieren a beneficios monetarios asociados con este pago de regalías.

Semejante situación contrasta con lo reportado en algunas localidades del Istmo de Tehuantepec donde las empresas hicieron aportes en especie (material, infraestructura) a escuelas, clínicas y centros comunitarios para llegar de manera indirecta a las mujeres. Es claro que estas acciones de «lavado verde» del extractivismo no logran compensar los daños generados (Escalón y González, 2017). Además, suelen ser apoyos que reproducen estereotipos de género en el sentido de que se asume que las mujeres son las responsables de la educación y salud de sus familias, con el resultante alargamiento de sus jornadas de trabajo. Sea lo que fuere, lo que aquí se quiere resaltar es que en Zacatecas no se reportó ninguna iniciativa que tratara de acercarse a las mujeres. Quizá esto se debe a la ausencia de movimientos de protesta contra la industria eólica en el estado. 


\section{Impactos en el uso y el costo de la energía por parte de las mujeres}

Es importante preguntarse hasta dónde llegan los impactos y beneficios de la transición energética cuando la forma que ésta adopta es la inversión privada en territorios rurales y marginados. Desde la perspectiva de género también es conveniente penetrar al interior de los hogares para dilucidar cómo viven las mujeres la responsabilidad de sostener la vida, y qué medios o beneficios reciben para que esto no represente una carga. Nuestro punto de entrada al ámbito doméstico fueron los usos y costos de la energía. No nos sorprendió descubrir que las mujeres sigan utilizando energéticos convencionales. Por muchos años, la leña ha sido el combustible más utilizado en los hogares mexicanos. Zacatecas no fue la excepción. En todas las localidades de estudio es común escuchar la palabra «tenamaste», vocablo nahua que se utiliza para describir un conjunto de piedras colocadas en forma circular que conforman un fogón comúnmente utilizado para preparar alimentos desde tiempos prehispánicos. Difícil no pensar en los paralelos entre el tenamaste y el comizcal, horno prehispánico que las mujeres del Istmo utilizan para hacer tortillas de maíz en medio de la invasión de aerogeneradores que caracteriza al Istmo de Tehuantepec (Vázquez et al., 2020).

En Zacatecas algunas mujeres siguen cocinando con leña. En toda la muestra se reportó la existencia de 30 estufas de leña (77\% de ellas en zonas rurales) y sólo seis han incorporado una chimenea que saca el humo fuera de la cocina. Aquí un testimonio sobre el daño que esto ocasiona: «uno hasta se enferma de los pulmones o los riñones porque ya recibió uno mucha humareda» (Estela Duarte, ama de casa). El problema del humo 
está presente en la cocina y en el baño. El boiler de leña tiene la ventaja de que no gasta gas, pero «hay que estarle atizando y ya cuando se bañó uno, sigue uno atizando, ya queda uno todo humeado. Ya ni se salvó la bañada» (participante anónima, Taller Benito Juárez).

El cuadro 1 muestra que el uso de leña es más común en el medio rural encuestado. También refleja el alcance que puede tener la promoción de FRE en el nivel doméstico gracias al trabajo de IZAC y el gobierno municipal de Judit Guerrero Torres (periodo 2017-2018) que promovieron calentadores solares de autoconstrucción o los proporcionaron subsidiados, al grado de que entre un tercio y la mitad de los hogares ya los utilizan (para mayor detalle, véase Vázquez y Sosa, 2019) con énfasis en las relaciones de género intergeneracionales que se establecen en torno a ellas. Los datos fueron recolectados en Zacatecas a través de una metodología mixta que involucró entrevistas, cuestionarios, grupos focales y devolución de resultados. Se utilizaron dos ejes de análisis: el contraste rural/urbano y la interseccionalidad de género. Las hipótesis fueron dos.

La combinación leña/gas que se presenta de manera predominante en el medio rural responde a tres factores. Primero, las mujeres de la tercera edad que usaron leña cuando eran jóvenes siguen recurriendo a ella porque es fácil de obtenerla cerca de casa: «Ya se llenaron los cerros de leña» (María Teresa Rodríguez, ama de casa). Un segundo motivo para seguir usando leña es el sabor de algunos alimentos: «La comida sabe más buena» (María Teresa Rodríguez, ama de casa). El tercero es el costo. El gasto promedio de gas al mes por hogar encuestado es de $\$ 351.81$ y la mayor parte de las mujeres (89.3\%) lo consideran caro: «Con el gas pues guisamos con más rapidez (...) La desventaja es que el gas es muy caro» (participante anónima, Taller Benito Juárez). 


\section{Cuadro 1}

Principales combustibles utilizados para cocinar

y calentar agua (porcentaje)

\begin{tabular}{|c|c|c|c|c|c|c|}
\hline $\begin{array}{c}\text { Tipo } \\
\text { de localidad/ } \\
\text { combustible }\end{array}$ & Rural & $\begin{array}{l}\text { Urbana } \\
\text { Cocina }\end{array}$ & Periurbana & Rural & $\begin{array}{l}\text { Urbana } \\
\text { Baño }\end{array}$ & Periurbana \\
\hline Gas y leña & 43.4 & 7.5 & 6.1 & 3.8 & 5.0 & 0.0 \\
\hline Únicamente gas & 56.6 & 90.0 & 93.9 & 34 & 35 & 45.1 \\
\hline Energía solar & 0.0 & 0.0 & 0.0 & 50.9 & 47.5 & 39.3 \\
\hline Electricidad & 0.0 & 2.5 & 0.0 & 7.5 & 12.5 & 12.1 \\
\hline Otro & 0.0 & 0.0 & 0.0 & 3.8 & 0.0 & 3.0 \\
\hline Total & 100 & 100 & 100 & 100 & 100 & 100 \\
\hline
\end{tabular}

Fuente: elaboración propia a partir de encuesta, n: urbana 40, periurbana 66 , rural 53.

Con relación a la electricidad, hay patrones de uso bastante más homogéneos. La mayoría (99.4\%) de los hogares encuestados cuentan con el servicio; los únicos que no lo tienen son los de Ojo de Agua de Meléndez, comunidad rural de difícil acceso. Las fallas en el servicio no son frecuentes; sólo se presentan en caso de eventos inusuales como lluvias o vientos fuertes. El gasto promedio bimestral de electricidad es de $\$ 290.39$ y la cantidad de mujeres que consideran caro el servicio de electricidad es de $46.5 \%$, notablemente menor en comparación con el gas.

No obstante, algunas personas expresaron dificultades para pagar el recibo de electricidad. En general pertenecen a la tercera edad que al momento de la entrevista recibían mil 160 pesos bimestrales del Programa Pensión para Adultos Mayores (PPAM). La señora Carmen Bustamante, 
ama de casa de la tercera edad, los aprovecha para sus gastos de gas, luz, «y ahí se acabaron». Un minero jubilado que usa tanque de oxígeno para sobrevivir está en problemas por la energía que consume: «Me está saliendo en 470 [pesos el recibo], pero pues digo yo, si no me conecto, pues ya se acabó Rafael (...) aunque me cueste, pero aquí estoy» (Rafael Pérez, minero jubilado). Al señor José Luis Guillén, también minero jubilado, le «sale más barata la luz» porque la utiliza poco: «Yo casi no prendo luces para nada».

En una situación donde la única forma de ahorrar electricidad es no prender la luz, resulta impensable instalar calefacción en las viviendas a pesar de que los cambios de temperatura pueden ir de $22^{\circ} \mathrm{C}$ en primavera-verano hasta $-11^{\circ} \mathrm{C}$ en el invierno. Así lo señaló Rafael: «Hace dos semanas cómo estaban los frillazos que se vinieron, y ahorita de repente se quita el frío y se suelta el calorón. Eso pues ya para uno que ya tiene edad, le hace más daños» (Rafael Pérez, minero jubilado).

De las mujeres encuestadas 10.7\% dijeron usar electricidad para calentar agua para el baño. La forma de hacerlo es introducir una plancha al agua: «Ya ves que la plancha tiene el fierrito de plancha, pues una que ya esté descompuesta, que no sirva, le quitas todo lo que es plástico y dejas el puro fierrito. Eso conectado, lo metes al agua» (Alma Guzmán, ama de casa). Esta forma tan peligrosa de calentar agua predomina entre hogares con muchos hijos e hijas o adultos mayores que no pueden costear el gas. No es una solución viable porque también aumenta el costo de la luz: «Se gasta muchísima luz. No hay dinero para pagar tanta luz. Y luego, tarda en calentarse también, y se gasta más luz. Cuando es un familionón, imagínese nomás, ¿cómo saldrá el recibo?» (participante anónima, Taller Benito Juárez).

Vale la pena preguntarse por qué la generación de energía del PEB no atiende, al menos en parte, la problemática de pobreza energética presente 
en estas comunidades. A pesar de que el nivel de cobertura de la red eléctrica es bueno, como también lo es en el resto del país ( $98 \%$ a escala nacional), las tarifas de electricidad resultan costosas para al menos la mitad de la población mexicana (Martínez y Gómez, 2017). La gente del municipio de Zacatecas no percibe ningún beneficio de la presencia del PEB en su territorio, como tampoco lo hace de otras políticas públicas destinadas a la eficiencia energética tales como la implementación del horario de verano: «Nos cobra el gobierno lo mismo (...) como el cambio de horario (...) gasta uno más (...) A nosotros nunca nos ha salido un recibo (...) más barato» (Rafael Pérez, minero jubilado).

En las comunidades cercanas al PEB la reducción en los costos de la electricidad podría contribuir a reducir la pobreza energética: «Que nos saliera un poquito más económico [el recibo de luz]» (Mauricio López, agricultor). Este tipo de beneficio debió de haber sido negociado por los ejidatarios firmantes del contrato desde el inicio del proceso:

Estaba diciendo el ingeniero (...) el mero perrón de ahí arriba, que cada turbina de esas daba energía para más de mil casas (...) pero fíjese para dónde van a echar la energía, para Puebla, allá para la Volkswagen, en lugar de aquí (...) A mí me aconseja un compa que tengo, estuvimos juntos en la escuela y es licenciadillo, pues ellos saben más (...) Dice, en el contrato hubieran dicho ustedes, queremos que nos dejen un transformador para que nos abastezca a El Orito y a Picones, eso hubiéramos hablado antes del contrato ahí (...) todavía pueden abran un juicio, todavía pueden nomás que sí póngase bien las pilas (Mauricio López, agricultor). 
Ninguna cláusula de estas características ha sido añadida a los contratos de energía eólica en México, a pesar de que la reducción en tarifas de luz es una de las demandas más sentidas del movimiento en contra de la expansión de la energía eólica en el Istmo de Tehuantepec. Esto se debe a que al capital privado no le interesa repartir beneficios, sino generar la mayor ganancia posible, y a que el Estado ha claudicado en su responsabilidad de proteger el interés de la ciudadanía. Sólo en Juchitán se logró una reducción en la tarifa de electricidad gracias a la movilización ciudadana en torno a la consulta normada por el Convenio 169 de la Organización Internacional del Trabajo (OIT) que estipula que los pueblos originarios deben participar en la toma de decisiones sobre inversiones que afectan su territorio (Chaca 2015; 2019).

Al no pertenecer a ningún pueblo originario, las comunidades del municipio de Zacatecas están en mayores dificultades para obtener beneficios como el de Juchitán. La ex presidenta municipal de Zacatecas Judit Guerrero López comentó en entrevista que durante su periodo de gobierno ella intentó negociar que la empresa pagara parte del recubrimiento asfáltico de la carretera que entra a El Orito. La negociación fue infructuosa a pesar de que en las localidades del Istmo las empresas generalmente responden a la protesta ciudadana con inversión en obra pública, particularmente pavimentación de vías de tránsito que de todas formas son necesarias para la construcción y mantenimiento del parque (Martínez y Gómez, 2017). Quizás la diferencia es que en Zacatecas no se registró ese tipo de protesta. 


\section{Reflexiones finales. Industria eólica, ¿desarrollo para quién?}

Este trabajo se propuso analizar los impactos y beneficios de la transición energética para los habitantes de comunidades rurales y marginadas del municipio de Zacatecas. La energía eólica tiene la capacidad de reducir la quema de combustibles fósiles, por lo que se ha convertido en una de las herramientas más importantes del combate al cambio climático. Sin embargo, hasta el día de hoy la propuesta se ha quedado al nivel de lo tecnológico, sin transformar el modelo de gobernanza bajo el cual es implementada. El artículo examina las implicaciones de género de la mercantilización del viento, entre ellas la exclusión en procesos de toma de decisiones, uso del territorio, reparto de beneficios y aprovechamiento de energía renovable.

Los grupos más desfavorecidos en este tipo de iniciativas suelen ser aquellos que desde antes de la llegada del proyecto ya tenían limitado su acceso a espacios de toma de decisiones y aprovechamiento de recursos naturales y productivos. En el caso del PEB, las personas excluidas fueron ejidatarios cuyos terrenos no formaron parte del polígono propuesto por la empresa; mujeres sin derechos de propiedad; personas de edad avanzada con ingresos precarios. El único beneficio identificado fue el económico y este se concentró en pocas manos, mayoritariamente masculinas. Puede decirse que los usuarios de distintos espacios de socialización (escuelas, clínicas, centros comunitarios) también fueron excluidos. Una misma persona puede compartir una o más de estas características de exclusión para dar lugar a grupos particularmente afectados. En otras palabras, aunque la industria eólica contribuye a la reducción de la quema de combustibles fósiles, deja mucho que desear en lo referente a la justicia social y de 
género para los y las usuarias del territorio, en particular los grupos más desfavorecidos entre los cuales se encuentran las mujeres.

Otra paradoja de la energía eólica, según se utiliza en México, es que constituye uno de los íconos del desarrollo modernizador más persuasivos de la época, que sin embargo se instala en regiones donde las mujeres usan herramientas prehispánicas para elaborar alimentos, o calientan agua para el baño introduciendo una plancha rota en la misma porque su familia es muy grande y no tienen forma de pagar el gas. Tanto en Zacatecas como en Oaxaca se identificó este contraste entre tradición y modernidad que atraviesa los cuerpos, la salud, los roles y las perspectivas de vida de las mujeres. Esto no quiere decir que estas sean enemigas del progreso. La adopción positiva de boilers solares en las comunidades de Zacatecas muestra que toda intervención tecnológica debe poner por delante las necesidades de la población para ser efectiva, participativa y de largo plazo.

Se concluye que la industria eólica comparte con otras iniciativas del modelo extractivista la priorización abstracta de la sostenibilidad global a expensas del incremento de las desigualdades sociales a nivel local. Lo que la hace distinta de otras actividades extractivas (por ejemplo, la megaminería) es su supuesta benignidad ambiental, pero lo cierto es que la incapacidad de involucrar a la población en los beneficios de la transición energética representa una enorme limitante de la industria eólica. Este problema se repite en cada lugar a donde llega, independientemente de su patrimonio biocultural, situación que obliga incluso a cuestionar los motivos por los cuales es necesaria la transición energética. Si el tan esperado cambio va a consistir en sacrificar la vocación agropecuaria de un lugar para beneficio de dos empresas (la que produce energía y la que la consume), habrá quienes digan que la búsqueda de la sustentabilidad no vale la pena. 
Desde la perspectiva de género, es necesario añadir a este planteamiento, que la tecnología que no penetra en los hogares y ayuda a resolver las necesidades más básicas de energía de las mujeres no puede considerarse una alternativa viable de desarrollo.

\section{Referencias}

Asesoría Ambiental Integral (s/f). «Documento Técnico Unificado del Trámite de Cambio de Uso de Suelo Forestal Modalidad B. Particular del Proyecto Denominado Proyecto Eólico MPG La Bufa». Zacatecas: Asesoría Ambiental Integral.

Asociación Mexicana de Energía Eólica (2018). «Asociación Mexicana de Energía Eólica». Recuperado de https://amdee.org/

Avilés Hernández, O.V. (2018). «Conflictos territoriales y perspectivas de desarrollo de la energía eólica en el istmo de Tehuantepec». Revista Mexicana de Opinión Pública (9), pp. 67-79. DOI: https://doi.org/10.22201/ fcpys.24484911e.2010.9.41818

Castillo Jara, E. (2011). «Problemática en torno a la construcción de parques eólicos en el Istmo de Tehuantepec». Revista Desarrollo Local Sostenible, 4(12), pp. 1-14. Recuperado de www.eumed.net/rev/delos/12

Chaca, R. (noviembre de 2015). «Guendaracane, descuentos de CFE por instalación de eólica en el Istmo». Quadratín.

Chaca, R. (mayo de 2019). «Inauguran en Juchitán el parque eólico más grande de América Latina». El Universal Oaxaca.

Comisión Nacional para el Diálogo con los Pueblos Indígenas (2013). «La energía eólica en México. Una perspectiva social sobre el valor de la tierra». Comisión 


\section{EXTRACTIVISMO, GÉNERO Y ENERGÍA EÓLICA EN EL MUNICIPIO DE ZACATECAS}

Nacional para el Diálogo con los Pueblos Indígenas. Recuperado de https:// docplayer.es/50047926-La-energia-eolica-en-mexico-una-perspectivasocial-sobre-el-valor-de-la-tierra.html

Composto, C. y Navarro, M.L. (2014). «Claves de lectura para comprender el despojo y las luchas por los bienes comunes naturales en América Latina». En Composto, C. y Navarro, M.L. (eds.), Territorios en disputa. Despojo capitalista, luchas en defensa de los bienes comunes naturales y alternativas emancipatorias para América Latina (pp. 33-75). México: Bajo Tierra Ediciones/ Jóvenes en Resistencia Alternativa.

Corscadden, K., Wile, A. y Yiridoe, E. (2012). «Social license and consultation criteria for community wind projects». Renewable Energy, 44, pp. 392-397. DOI: https://doi.org/10.1016/j.renene.2012.02.009

Quintana, D. y Serafín R. (2014). «Redes de pesca y redes de poder. La lucha de Los pescadores de San Dionisio Del Mar por su gobernanza, territorialidad y autonomía». En Novelo, F. (ed.), Instituciones y Desarrollo (pp. 589-624). Ciudad de México: Universidad Autónoma Metropolitana.

Quintana, D. y Serafín R. (2015). «Energía limpia o energía perversa: actores sociales y parques eólicos en Dinamarca y en el Istmo de Tehuantepec». En Corona, J.M. (ed.), Desarrollo sustentable: enfoques, políticas, gestión y desafíos (pp. 517-538). Ciudad de México: Universidad Autónoma Metropolitana.

Quintana, D. y Serafín R. (2016). «La disputa por los bienes de la nación mexicana: megaproyectos vs comunidades». Revista Alternativa (5), pp. 88-113.

Dunlap, A. (2018). «The ssolution` is now the 〈problem»: wind energy, colonisation and the «genocide-ecocide nexus〉 in the Isthmus of Tehuantepec, Oaxaca». International Journal of Human Rights, 22(4), pp. 550-573. DOI: https:// doi.org/10.1080/13642987.2017.1397633. 
Durand, L., Nygren, A. y De la Vega-Leinert, A.C. (2019). «Introducción». En Durand, L. (ed.), Naturaleza y neoliberalismo en América Latina (pp 570). Cuernavaca: Centro Regional de Investigaciones Multidisciplinarias/ Universidad Nacional Autónoma de México.

Escalón Portilla, E. y González Gaudiano, E. (2017). «La escuela como actor social en las luchas contra el extractivismo. Prácticas político-pedagógicas desde la educación comunitaria en Oaxaca, México». Diálogos sobre educación. Temas actuales en investigación educativa, 8(15), pp. 1-28.

García-Torres, M. (2018). El IBEX 35 en guerra contra la vida. Un análisis ecofeminista. Madrid: Ecologistas en Acción.

Gay-Antaki, M. (2016). «Now we have equality): a feminist political ecology analysis of carbon markets in Oaxaca, Mexico». Journal of Latin American Geography, 15(3), pp. 49-66.

Gudynas, E. (febrero de 2013). «Extracciones, extractivismos y extrahecciones. Un marco conceptual sobre la apropiación de recursos naturales». Observatorio del Desarrollo (18), pp. 1-17. Recuperado de http://ambiental.net/ wp-content/uploads/2015/12/GudynasApropiacionExtractivismoExtraheccionesOdeD2013.pdf

Honty, G. y Gudynas, E. (2014). Cambio climático y transiciones al Buen Vivir: alternativas al desarrollo para un clima seguro. Lima: Centro Latinoamericano de Ecologia Social (CLAES). DOI: https://doi.org/10.1017/ CBO9781107415324.004.

Howe, C. y Dominic B. (2016). «Aeolian extractivism and community wind in Southern Mexico». Public Culture, 28(279), pp. 215-235. DOI: https://doi. org/10.1215/08992363-3427427 


\section{EXTRACTIVISMO, GÉNERO Y ENERGÍA EÓLICA EN EL MUNICIPIO DE ZACATECAS}

Instituto Nacional de Estadística y Geografía (INEGI) (2015). "Capa de municipios de la República mexicana 1:250000». Recuperado de conabio.gob.mx/ información/gis/

Juárez-Hernández, S. y León, G. (2014). «Energía eólica en el Istmo de Tehuantepec: desarrollo, actores y opisición social». Problemas del Desarrollo, 178(45), pp. 139-162. DOI: https://doi.org/10.1016/S0301-7036(14)70879-X.

León, I. (noviembre de 2017). «Tras 7 años, Power Group inaugurará parque eólico en Zacatecas». El Financiero.

Levy Gálvez, K. et al. (2015). «Informe del grupo asesor de las organizaciones de la sociedad civil en la consulta indígena en Juchitán de Zaragoza para la instalación de un parque eólico para la generación de 396 MW de energía eléctrica». Juchitán de Zaragoza: Consulta Indígena. Recuperado de https:// consultaindigenajuchitan.files.wordpress.com/2015/07/informe-del-grupoasesor.pdf

López Gómez, A.L. (2015). «Cambio climático y conflictos ecológico distributivos en regiones indígenas de México. El caso de la industria eólica en el Istmo de Tehuantepec, Oaxaca». Barcelona: Universidad Autónoma de Barcelona. Machado Aráoz, H. (2015). «Horacio Machado Aráoz». Bajo el volcán, 15(23), pp. 1151. Recuperado de https://www.redalyc.org/articulo.oa?id=28643473002

Martínez Mendoza, E., Rivas Tovar, L.A. y Vera Martínez, P.S. (2018). «El sector eólico en México y España». Perfiles Latinoamericanos, 27(53), pp. 1-21. DOI: https://doi.org/10.18504/pl2753-002-2019

Martínez, M. y Gómez, A. (2017). «Vientos del capitalismo verde: globalización, desarrollo y transición energética en el Istmo de Tehuantepec (Oaxaca, México)». Ciencia \& Trópico, 41(1), pp. 15-54.

México Power Group (2014). «México Power Group». México Power Group. http://mexicopowergroup.com/index.php/es/ 
Nahmad, S., Nahón, A. y Langlé, R. (2014). La visión de los actores sociales frente a los proyectos eólicos del Istmo de Tehuantepec. Oaxaca: Consejo Nacional de Ciencia y Tecnologia/Centro de Investigaciones y Estudios Superiores en Antropología Social.

Okkonen, L. y Lehtonen, O. (2016). «Socio-economic impacts of community wind power projects in Northern Scotland». Renewable Energy, 85, pp. 826833. DOI: https://doi.org/10.1016/j.renene.2015.07.047.

Olivera, B. (2019). «La participación de las mujeres rurales en la defensa del territorio en México». En Del Pozo Martínez, E. y Martínez, J.C. (eds.), Caminos desde abajo. Organización social y usos del Derecho en la defensa del territorio en México (pp. 247-279). Ciudad de México: Fundar/Centro de Análisis e Investigación/Gedisa.

Penna-Firme, R. (2015). «Green» markets and the appropriation of land». Ciência Geográfica-Bauru, XIX(1), pp. 109-118.

Sánchez Fonseca, M. (2008). «Energía eólica en el Istmo de Tehuantepec y sus impactos socioambientales». Ciudad de México: Universidad Autónoma Metropolitana.

Simcock, N. (2014). «Exploring how stakeholders in two community wind projects use a «those affected principle to evaluate the fairness of each project's spatial boundary». Local Environment, 19(3), pp. 241-258. DOI: https://doi.org /10.1080/13549839.2013.788482

Tejada, L. y Rist, S. (2019). «El boom del bioetanol y (re)concentración de la tierra en la costa norte peruana: luchas agrarias en un contexto neoliberal». En Durand, L., Nygren, A. y De la Vega-Leinert, A.C. (eds.), Naturaleza y neoliberalismo en América Latina. Cuernavaca: Centro Regional de Investigaciones Multidisciplinarias/Universidad Nacional Autónoma de México. 
Torres Fragoso, J. y Luna Espinoza, I. (2018). «Políticas públicas para el desarrollo regional el caso de la industria eólica en el Istmo de Tehuantepec, Oaxaca, México», Ciencia Administrativa (1), pp. 125-146.

Trench, T. y Libert Amico, A. (2019). «EEs REDD+ un instrumento de neoliberalización? Experiencias desde Chiapas y Yucatán en el sur de México». En Durand, L., Nygren, A. y De la Vega-Leinert, A.C. (eds.), Naturaleza y neoliberalismo en América Latina. Cuernavaca: Centro Regional de Investigaciones Multidisciplinarias/Universidad Nacional Autónoma de México.

Vázquez García, V., Martínez Reyes, S.N., Pérez Olvera, M.A. y Rueda Abad, J.C. (2020). «De zapalote chico, chahuiteros y totoperas. Género, etnia y maíz en el istmo de Tehuantepec, México». LEISA Revista de Agroecología, 36(1), pp. 18-20.

Vázquez García, V. y Sosa Capistrán, D.M. (2019). «Seguridad energética e interseccionalidad de género en Zacatecas, México». Sociedad y Ambiente (21), pp. 131-154. DOI: https://doi.org/10.31840/sya.v0i21.2043. 